\title{
OPTION PRICING AND THE DIRICHLET PROBLEM
}

MARK S. JOSHI

\section{INTRODUCTION}

Laplace's equation is ubiquitous in physics: it arises in the study of many areas such as electromagnetism, gravity and fluid dynamics since it can be used to describe the behaviour of electric, gravitational and fluid potentials. If we take a body with prescribed temperature on the boundary, then the long-term equilibrium temperature in the interior also satisfies Laplace's equation with Dirichlet boundary conditions, that is the value of the solution on the edge is the prescribed temperature.

Despite, the equation's importance in physics, it has not been important so far in finance. Here, we will relate it to options' pricing. It is well-known that the Dirichlet problem for the Laplacian on a reasonably smooth compact domain in $\mathbb{R}^{n}$ can be solved using Brownian motion. Indeed the result was found by Kakutani in 1944, [3, 4]. In this note, I want to discuss how this result can be reinterpreted financially. Our objective is to increase our intuition about the problem rather than to attempt to prove new results. We will therefore work in a restricted case rather than in the most general case possible to keep technicalities minimal. For more general cases, and a more rigorous treatment not involving finance, see [5]. We will also examine the time-reversed heat equation where the connections are with the Feynman-Kac theorem, [1], [2] and [6].

Let $\Omega$ be an open bounded subset of $\mathbb{R}^{n}$ with compact closure, $\bar{\Omega}$. We assume that $\partial \Omega$ is smooth in the sense that it is an embedded submanifold. Given a reasonable function, e.g. a continuous function, $f$ from $\partial \Omega$ to $\mathbb{R}$ the Dirichlet problem for the Laplacian is to find a function $u$ which is continuous on $\bar{\Omega}$, such that

$$
\begin{gathered}
u_{\mid \partial \Omega}=f, \\
\Delta u=0,
\end{gathered}
$$

Key words and phrases. option pricing, Dirichlet problem, maximum principle. 
where

$$
\Delta u=\sum_{j=1}^{n} \frac{\partial^{2} u}{\partial x_{j}^{2}} .
$$

To solve this problem via Browian motion, one defines $u(x)$ by the expected value of $f$ at the first time a Brownian motion starting at $x$ reaches $\partial \Omega$. We will show here that this solution has a natural financial interpretation, in terms of the equivalence of the martingale and PDE approaches to pricing derivatives.

\section{THE FINANCIAL INTERPRETATION}

Let $X_{i}$ for $i=1, \ldots, n$, be non-dividend paying financial assets following independent Brownian motions. We also assume the existence of riskless bond, $B$, of constant value 1 . We shall use $B$ as numeraire. Let $C$ denote a product with no expiry that pays a continuous function $f$ at the first time the vector $\left(X_{1}, \ldots, X_{n}\right)$ touches $\partial \Omega$.

We are in a complete market with zero interest rates so the price of $C$ will be a function of $\left(X_{1}, \ldots, X_{n}\right)$ with no $t$ - dependence since there is no expiry. How can we price this option? If we proceed with the PDE approach. We can compute

$$
d(C(X))=\frac{1}{2} \sum_{j=1}^{n} \frac{\partial^{2} C}{\partial x_{j}^{2}} d t+\sum_{j=1}^{n} \frac{\partial C}{\partial X_{j}} d X_{j} .
$$

The usual hedging arguments allow us to eliminate the Brownian term, and since there are no interest rates, we conclude

$$
\Delta u=0,
$$

with the obvious boundary condition $C_{\mid \partial \Omega}=f$. So $C(x)$ solves the Dirichlet problem for the Laplacian.

Now suppose we try the martingale approach. Let $\tau$ be the first time of hitting the boundary then $\tau$ will be a stopping time with finite expectation, since $\Omega$ is bounded. The value of $C$ will be equal to the expectation at time $\tau$. So

$$
C(X)=C_{0}(X)=\mathbb{E}\left(C_{\tau}(X)\right) .
$$

But $C_{\tau}(X)$ is just the value of $f$ at the point where $X$ first touches the boundary. The price of $C$ at $x$ is therefore the expectation of the value of $f$ at the time of first boundary touching for a Brownian motion starting at $x$.

Putting the two interpretations together, we have the result we set out to prove. 


\section{No ARBitrage AND the MAXIMUM PRINCIPLE}

If $f$ is constant then the solution $u$ is equal to the same constant everywhere and life is rather boring. We therefore assume that $f$ is nonconstant with minimum $m$ and maximum $M$. The maximum principle states that the minimum and maximum of $u$ are $m$ and $M$, respectively, and that these values are attained only on the boundary.

At an interior point $x$, consider the value of $C(x)$. The most $C$ will pay is $M$ and the minimum $C$ will pay is $m$, (since it will hit the boundary with probability one in finite time.) Its value must therefore lie between $m$ and $M$, by no-arbitrage. If it has the actual value $M$, then consider the portfolio of $M$ bonds minus one unit of $C$. This portfolio has zero initial value. However, if $f$ is non-constant it has non-zero probability of finishing at a point where $f(x)<M$. We conclude that either $f$ is constant or there is an arbitrage. Thus for non-constant $f$, the maximum of $C$ cannot be obtained in $\Omega$. Similarly, for the minimum of $C$.

\section{The HeAT EQUATION}

Now suppose that instead of the option having infinite expiry, a rebate is paid if the boundary is not reached by time $T$. We let the rebate vary according to the value of $X$ at time $T$. It therefore pays a continuous function $g$ on $\bar{\Omega}$. For concreteness, we require $f$ to agree with $g$ on $\partial \Omega$.

The value of the option $C$ will now be time-dependent, and will have a price $C(t, X)$. We have the obvious boundary conditions.

$$
\begin{aligned}
C(T, X) & =g(X), \\
C(t, X) & =f(X), \text { for } \quad X \in \partial \Omega .
\end{aligned}
$$

We now have

$$
d(C(X, t))=\left(\frac{\partial C}{\partial t}+\frac{1}{2} \sum_{j=1}^{n} \frac{\partial^{2} C}{\partial x_{j}^{2}}\right) d t+\sum_{j=1}^{n} \frac{\partial C}{\partial X_{j}} d X_{j} .
$$

After hedging the Brownian part, we are left with

$$
\frac{\partial C}{\partial t}+\frac{1}{2} \sum_{j=1}^{n} \frac{\partial^{2} C}{\partial x_{j}^{2}}=0
$$

This is the time-reversed heat-equation.

What about the martingale approach? Let $\tau$ be the minimum of the time of first hitting $\partial \Omega$ and time $T$. The pay-off is then the value of $C$ at time $\tau$ which will be $g(X)$ if $\partial \Omega$ has not been reached and $f(X)$ 
otherwise. The stopping time $\tau$ is finite and there are no interest rates, so we have

$$
C(X, t)=\mathbb{E}_{t, X}\left(C\left(X_{\tau}, \tau\right)\right)
$$

In other words, the value of $C$ at time $t$ at point $X$ is the expectation of its value on exiting the domain $\Omega \times(t, T)$ by either hitting the boundary, $\partial \Omega$, or $\Omega \times T$.

Putting our two pricing approaches together, we therefore conclude that the solution to the time-reversed heat equation can be obtained via an expectation over a Brownian motion. If we want to solve the heat equation itself, we simply put $t=-s$.

Let $m$ be the minimum of $f(x)$ and $g(x)$ over their domains, and similarly for $M$ and the maximum. If $m \neq M$, we can prove similarly as for the Laplacian that the solution of the heat equation lies strictly between $m$ and $M$ everywhere in $\Omega \times(0, T)$. In particular, taking the portfolio $P=M B-C$ where $B$ is the riskless bond, together with the reinvestment rule that all pay-offs are used to buy riskless bonds, we have that $P$ is of non-negative and possibly positive value at time $T$, since there is non-zero probability of reaching a point where the pay-off is $M$ and also of reaching a point where the pay-off is less than $M$. It must therefore be of positive value at any time $t<T$ by no-arbitrage, we conclude that

$$
C(t, X)<M
$$

for $t<T$ and $X \in \Omega$. The portfolio

$$
P^{\prime}=C-m B,
$$

yields $m<C(t, X)$, giving us both the maximum and the minimum.

\section{CONCLUSiON}

We have not proven any new results, however, we have seen that a classical technique has a natural interpretation in terms of derivatives pricing. With this interpretation the maximum principle becomes rather obvious. We have studied a rather simple special case, the flat Laplacian on $\mathbb{R}^{n}$, however, it is clear that the techniques could easily be applied in a much wider range of cases.

\section{REFERENCES}

[1] R. P. Feynman, Space-time approach to non-relativistiv quantum mechanics, Rev. Mod. Phys, 20, 367-387, 1948.

[2] M. Kac, On distributions of certain Wiener functionals, Trans. Amer. Math. Soc., 65, 1-13, 1949

[3] S. Kakutani, On Brownian motion in n-space, Proc. Acad. Japan, 20, 648-652, 1944 
[4] S. Kakutani, On two-dimensional Brownian motion and harmonic functions, Proc. Acad. Japan, 20, 706-714, 1944

[5] I. Karatzas, E. Shreve, Brownian Motion and Stochastic Calculus, Second edition, Springer Verlag, 1997

[6] M. Rosenblatt, On a class of Markov processes. Trans. Amer. Math. Soc. 71, (1951). 120-135.

Centre for Actuarial Studies, Department of Economics, University of Melbourne, Victoria 3010, Australia

E-mail address: mark@markjoshi.com 


\section{University Library}

\section{- M M N E R VA A gateway to Melbourne's research publications}

Minerva Access is the Institutional Repository of The University of Melbourne

Author/s:

JOSHI, M

Title:

Option pricing and the Dirichlet problem

Date:

2006

Citation:

JOSHI, M. (2006). Option pricing and the Dirichlet problem. Wilmott Magazine, 4 (4), pp. $100-103$

Persistent Link:

http://hdl.handle.net/11343/34308 